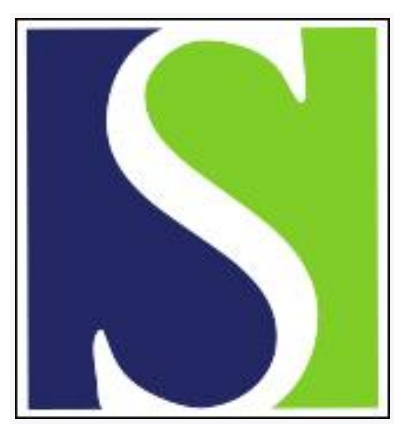

Scand J Work Environ Health 2001;27(6):412-419

https://doi.org/10.5271/sjweh.634

Issue date: Dec 2001

Alcohol-related hospital utilization and mortality in different occupations in Sweden in 1991-1995

by Hemmingsson T, Ringbäck Weitoft $\mathrm{G}$

Affiliation: Department of Occupational Health, Norrbacka, SE-171 76 Stockholm, Sweden. tomas.hemmingsson@smd.sll.se

Key terms: alcohol-related hospital utilization; alcoholism; female; long-term employment; male; mortality; occupation; selection; short-term employment

This article in PubMed: www.ncbi.nlm.nih.gov/pubmed/11800329 


\title{
Alcohol-related hospital utilization and mortality in different occupations in Sweden in 1991-1995
}

\author{
by Tomas Hemmingsson, PhD, ${ }^{1}$ Gunilla Ringbäck Weitoft, $B S c^{2}$
}

\begin{abstract}
Hemmingsson T, Ringbäck Weitoft G. Alcohol-related hospital utilization and mortality in different occupations in Sweden in 1991-1995. Scand J Work Environ Health 2001;27(6):412-419.
\end{abstract}

\begin{abstract}
Objectives This study investigated alcohol-related hospital utilization and alcohol-related mortality according to occupation among men and women. Whether increased rates of alcoholism in some occupations result from circumstances within the occupation or from selective recruitment of persons prone to alcohol misuse was studied.

Methods All Swedish residents were included who reported an occupation in the censuses of 1985 and 1990 and were born in 1926-1960. The relationships between occupation and hospitalization due to an alcoholism diagnosis in 1991-1994 and alcohol-related mortality in 1991-1995 were studied among stable workers (those who held the same occupation in both censuses) and newly recruited workers (those who held different occupations in the two censuses). Incidence and mortality rates were calculated for the different occupations using the person-year method, and standardized rate ratios were used as approximations of the relative risk of disease occurrence and mortality in different occupations as compared with the corresponding statistics of the entire study population.

Results Several, mostly manual, occupations showed an increased relative risk of alcoholism diagnoses and alcohol-related mortality. Nonmanual occupations had low risks. Women in male-dominated high-risk occupations often showed increased relative risks. Stable and newly recruited employees in the same occupation showed very similar relative risks.

Conclusions New recruits into high-risk occupations often have increased relative risks of at least the same magnitude as persons employed long-term in the same occupations. This finding indicates that the increased relative risk of alcoholism found in some occupations can partly be explained by selective recruitment of heavy drinkers.
\end{abstract}

Key terms alcoholism, females, long-term employment, males, selection, short-term employment.

Several studies have shown differing rates of alcoholism between occupations (1-8). It is often concluded that blue-collar occupations have higher rates of alcoholism when compared with white-collar occupations, while those outside the labor market are at the highest risk. Several explanations have been proposed for the rate of alcoholism being higher in some occupations than in others. The proposed explanations, as summarized by Martin et al (9), include (i) placement in stressful or unrewarding jobs, (ii) participation in job-based drinking networks, and (iii) absence of work-based social support.
However, it is not clear whether the increased rates of alcoholism in some occupations are related to circumstances within the occupation or whether they are a result of selection of heavy drinkers to the occupation (10).

In this study we analyzed alcoholism diagnoses given in inpatient care in 1991-1994 and alcohol-related mortality in 1991-1995 in relation to occupation, based on data from the national population and housing censuses in 1985 and 1990. We have also compared stable workers in each occupation (ie, those who reported the same occupation in 1985 and 1990) with those newly

1 Division of Occupational Health, Department of Public Health Sciences, Karolinska Institute, Stockholm, Sweden.

2 Center for Epidemiology, National Board of Health and Welfare, Stockholm, and Department of Public Health and Clinical Medicine, Umeå University, Umeå, Sweden.

Reprint requests to: Tomas Hemmingsson, Department of Occupational Health, Norrbacka, SE-171 76 Stockholm, Sweden. [E-mail: tomas.hemmingsson@smd.sll.se] 
recruited (ie, those who reported different occupations in the same two censuses). If long-term employed in some occupations show an increased rate of alcoholism while those newly recruited do not circumstances within the occupation could be considered important for the development of alcoholism. However, if an increased rate were found also among those who are newly recruited to the same occupation, selection would probably play a role.

The aims of this study were to investigate the relationship between occupation, based on the occupationally stable part of the workforce (ie, those men and women who reported the same occupation in the censuses in 1985 and 1990) and alcoholism diagnoses in 1991-1994, as well as alcohol-related mortality in 1991-1995.

In order to investigate the importance of selection, we compared the risk estimates of alcoholism diagnoses made in inpatient care for occupations in the occupationally stable part of the workforce with the risk estimates of new recruits to the same occupations (ie, those who reported different occupations in the two censuses).

\section{Subjects and methods}

\section{Study population}

Between 1960 and 1990 computer-based censuses were carried out in Sweden every fifth year. The censuses comprise information on age, gender, place of residence, current occupation, and current socioeconomic group. The population studied was all Swedish residents who reported an occupation in both the censuses of 1985 and 1990 and who were born between 1926 and 1960 .

\section{Outcome}

Information on alcoholism diagnoses was obtained from the computerized National Hospital Discharge Register, administered by the Center for Epidemiology at the National Board of Health and Welfare in Sweden. It has covered all public inpatient care in Sweden since 1987. The computer-based linkage was made possible by the unique personal identification number of every citizen in Sweden. The study population was followed with regard to alcohol-related disorders during the period 1991-1994. Information on the following outcomes was obtained: liver cirrhosis (International Classification of Diseases, 9th revision (ICD-9) code 571.0-571.3), alcohol psychosis (ICD-9 code 291), alcohol dependence (ICD-9 code 303), alcohol abuse (ICD-9 code 305.0), alcohol intoxication (ICD-9 code 980). The latter four diagnoses were analyzed together as "alcoholism diagnoses". For each individual the first relevant diagnosis at any discharge during the follow-up period was used for each outcome by checking primary as well as contributory diagnoses.

Data on mortality was obtained through individual linkage to the Swedish Cause of Death Register, which is maintained by the National Board of Health and Welfare. The study population was followed with regard to alcohol-related mortality during the period 1991-1995. In the analyses we used a previously defined construct including the following causes of death as the underlying or contributory cause of death (11): alcohol psychosis (ICD-9 code 291), alcoholism (ICD-9 code 303), alcohol abuse (ICD-9 code 305.0), alcohol polyneuropathy (ICD-9 code 357.5), alcohol-related cardiomyopathy (ICD-9 code 425.5), gastritis from alcohol (ICD-9 code 535.3), liver cirrhosis (ICD-9 code 571.0-571.3), and alcohol intoxication (ICD-9 code E860, E980, 980).

\section{Data on occupation}

The occupations reported in the censuses of 1985 and 1990 were coded according to the Nordic modification of the three-digit International Standard Classification of Occupations (NYK 1973). Those who reported the same occupation in the censuses of 1985 and 1990 were regarded as having long-term, or stable, employment. Those in an occupation in the 1990 census who reported being in another occupation in the 1985 census were regarded as having short-term, unstable, employment or as new recruits to the occupation. A distinction between manual (blue-collar) and nonmanual (white-collar) occupations was made according to the Swedish socioeconomic classification.

\section{Statistical methods}

Incidence rates and mortality rates were calculated for the different occupations using the person-year method. The number of person-years at risk was calculated by adding up the years the persons were alive during the period of follow-up. The year of death was counted as half a year for that specific calendar year. The standardized rate ratio (SRR) for hospitalization due to an alcoholism diagnosis or for mortality, with the $95 \%$ confidence interval $(95 \% \mathrm{CI})$, was used as an approximation of the relative risk of disease and mortality, respectively, in different occupations in a comparison with the study population as a whole. The computations were performed with allowance for age (5-year age categories), county ( 24 counties), and degree of urbanization (2 categories) with weights according to the distribution of the potential confounders in the entire study population. 
In the analysis, for each gender, only occupations were considered that had at least 20 observed or expected cases in the occupation.

\section{Results}

Table 1 shows that $23 \%$ of both the men and the women had been newly recruited into an occupation according to the 1990 census. Those newly recruited were 34 years younger than the stable group.

As shown in table 2, 13071 men and 3913 women with alcoholism diagnoses and 1926 men and 1156 women with liver cirrhoses diagnoses were identified during the follow-up period of 1991-1994. Altogether 20792 men and 11388 women died during the followup period of 1991-1995, whereof the causes were alcohol-related for 1849 men and 429 women.

\section{Alcoholism diagnoses in occupations}

Men. Among the men who held the same occupation in 1985 and 1990, 37 occupations with more than 20 cases, mainly for skilled and unskilled manual workers, showed significantly increased relative risks of alcohol diagnoses. The reference category was the entire study population (ie, those who held an occupation in both censuses) (table 3). Among the occupationally stable male workers (SRR1), five occupations had a relative

Table 1. Number (N) of persons in the study according to gender, status (short-term or long-term employment), and average age in 1990.

\begin{tabular}{|c|c|c|c|c|c|c|}
\hline & \multicolumn{6}{|c|}{ Type of employment } \\
\hline & \multicolumn{3}{|c|}{ Long-term } & \multicolumn{3}{|c|}{ Short-term } \\
\hline & $\mathrm{N}$ & $\%$ & $\begin{array}{l}\text { Average age } \\
\text { (years) }\end{array}$ & $\mathrm{N}$ & $\%$ & $\begin{array}{l}\text { Average age } \\
\text { (years) }\end{array}$ \\
\hline Men & 1079641 & 76.6 & 46.2 & 329701 & 23.4 & 42.4 \\
\hline Women & 961643 & 76.3 & 46.2 & 298321 & 23.7 & 43.0 \\
\hline
\end{tabular}

risk of 3.0 or more (horticulture workers, forest workers, sailors, smiths and forgers, other metal processing work, bakers), and 19 occupations had a relative risk of $\geq 2.0$. Social work was the only nonmanual occupation with an increased relative risk (RR 1.3, 95\% CI 1.0-1.7) among the men.

Among the men who were in the same occupation in both censuses, nine occupations had a relative risk of $\geq 3.0$ (horticulture workers, forest workers, messengers, butchers, unskilled manual workers, packers, kitchen assistants, home helpers, cleaners). For 21 occupations the risk was $\geq 2$ (table 3 ). Among the high-risk occupations, those recruited in 1990 often showed higher risk increases than the persons with stable occupations did. Two occupations, drivers and construction carpenters, did not show an increased relative risk among occupationally stable workers, but there was an increased risk among those in the occupation only in 1990.

Nonmanual occupations almost consistently showed low risks for alcoholism diagnosis (table 4). There were only very small relative risk differences between the persons in stable and unstable work in the low-risk occupations.

Women. Among the women increased relative risks were mainly found in manual occupations. Eleven occupations among the occupationally stable persons (SRR1) had significantly increased relative risks. Eight of these occupations also had increased relative risks among the men. Five occupations (tailors and sewers, toolmakers, machinery fitters, cooks, and cleaners) had a relative risk of $\geq 2.0$ (based on at least 20 cases) (table 3).

Table 3 shows that the women who entered high-risk occupations, defined from the occupationally stable part of the workforce, also showed increased relative risks during the follow-up (SRR2).

As for the men, occupations with low relative risks for alcoholism diagnosis were mainly nonmanual (table 4). Among the low-risk occupations there were only very small relative risk differences between the persons in stable and unstable work. The low-risk oc-

Table 2. Liver cirrhosis diagnosis in 1991-1994, alcoholism diagnoses in 1991-1994, alcohol-related mortality in 1991-1995, all-cause mortality in 1991-1995, and the number of person years.

\begin{tabular}{|c|c|c|c|c|}
\hline \multirow[b]{2}{*}{ Variable } & \multicolumn{2}{|c|}{$\begin{array}{c}\text { Men } \\
(\mathrm{N}=1409342)\end{array}$} & \multicolumn{2}{|c|}{$\begin{array}{c}\text { Women } \\
(\mathrm{N}=1259964)\end{array}$} \\
\hline & $\mathrm{N}$ & Person-years & $\mathrm{N}$ & Person-years \\
\hline Liver cirrhosis diagnoses (1991-1994) & 1926 & 5393482 & 1156 & 4845629 \\
\hline Alcoholism diagnoses (1991-1994) & 13071 & 5369162 & 3913 & 4839514 \\
\hline Alcohol related mortality (1991-1995) & 1849 & 6672550 & 429 & 5995697 \\
\hline All cause mortality (1991-1995) & 20792 & 6672550 & 1388 & 5995697 \\
\hline
\end{tabular}


Table 3. Standardized rate ratios (SRR) for the occupations with increased risk of alcoholism diagnoses among long-term employed men and women (who reported the same occupation in 1985 and 1990) and among newly recruited (ie, short-term employed) workers (those who reported the occupation only in 1990 and reported another occupation in 1985). The reference category was the entire study population (ie, those who reported an occupation in both censuses). Only occupations for which the lower limit of the $95 \%$ confidence interval $(95 \% \mathrm{CI}$ ) was $\geq 1.0$ based on at least 20 cases among men (stable or unstable in occupation) or women (stable or unstable in occupation) are listed. SRR values in bold indicate that the criteria was fulfilled. (nec $=$ not elsewhere classified)

\begin{tabular}{|c|c|c|c|c|c|c|c|c|c|c|c|c|}
\hline \multirow{3}{*}{ Occupation a } & \multicolumn{6}{|c|}{ Men } & \multicolumn{6}{|c|}{ Women } \\
\hline & \multicolumn{3}{|c|}{ Long-term employed } & \multicolumn{3}{|c|}{ Short-term employed } & \multicolumn{3}{|c|}{ Long-term employed } & \multicolumn{3}{|c|}{ Short-term employed } \\
\hline & $\mathrm{N}$ & SRR & $95 \% \mathrm{Cl}$ & $\mathrm{N}$ & SRR & $95 \% \mathrm{Cl}$ & $N$ & SRR & $95 \% \mathrm{Cl}$ & $\mathrm{N}$ & SRR & $95 \% \mathrm{Cl}$ \\
\hline Attendants in psychiatric care (NYK 042) & 67 & 2.4 & $1.8-3.1$ & 9 & 3.5 & $1.4-9.1$ & 42 & 1.9 & $1.4-2.7$ & 9 & 1.5 & $0.7-3.0$ \\
\hline Assistant nurses (NYK 043) & 85 & 2.7 & $2.1-3.5$ & 44 & 2.0 & $1.4-2.7$ & 344 & 1.4 & $1.3-1.6$ & 89 & 1.8 & $1.5-2.3$ \\
\hline Social workers (NYK 092) & 72 & 1.3 & $1.0-1.7$ & 31 & 1.5 & $1.0-2.2$ & 32 & 0.5 & $0.3-0.7$ & 15 & 0.6 & $0.4-1.1$ \\
\hline Horticultural workers (NYK 412) & 179 & 3.6 & $3.0-4.3$ & 64 & 6.3 & $3.7-10.9$ & 4 & 9.9 & $1.8-51.5$ & 6 & 4.2 & $1.7-10.4$ \\
\hline Forest workers, log drivers (NYK 441) & 254 & 8.8 & $6.8-11.3$ & 63 & 10.9 & $7.1-16.7$ & - & .. & .. & 1 & 2.7 & $0.4-19.5$ \\
\hline Sailors (NYK 611) & 52 & 3.5 & $2.2-5.4$ & 6 & 1.5 & $0.5-4.5$ & - & .. & .. & .. &.. & .. \\
\hline Drivers (NYK 633) & 529 & 1.0 & $0.9-1.1$ & 167 & 1.6 & $1.3-1.9$ & 20 & 1.9 & $1.2-3.1$ & 11 & 1.9 & $1.0-3.7$ \\
\hline Messengers (NYK 662) & 101 & 1.9 & $1.6-2.4$ & 68 & 3.2 & $2.3-4.3$ & 3 & 1.2 & $0.4-4.1$ & 1 & 0.2 & $0.1-1.5$ \\
\hline Railway linesmen (NYK 678) & 23 & 1.7 & $1.0-2.7$ & 9 & 2.1 & $1.0-4.7$ & - &.. & .. & .. & .. & .. \\
\hline Tailors and sewers (NYK 711) & 3 & 3.7 & $0.6-21.4$ & 1 & 0.9 & $0.1-6.6$ & 25 & 2.5 & $1.5-4.3$ & 12 & 3.3 & $1.4-7.8$ \\
\hline Smiths and forgers (NYK 736) & 29 & 3.0 & $1.3-7.2$ & 11 & 3.3 & $1.5-7.2$ & - & .. &. & - &.. & .. \\
\hline Metal processing work nec (NYK 738) & 24 & 4.0 & $1.6-9.9$ & 11 & 1.2 & $0.5-3.2$ & 1 & 0.1 & $0.1-1.0$ & - & .. & .. \\
\hline Toolmakers (NYK 750) & 482 & 1.7 & $1.6-1.9$ & 157 & 2.3 & $1.9-2.8$ & 34 & 2.0 & $1.2-3.3$ & 20 & 4.6 & $2.2-9.3$ \\
\hline Machinery fitters (NYK 751) & 485 & 1.1 & $1.0-1.2$ & 161 & 1.8 & $1.5-2.2$ & 30 & 2.7 & $1.7-4.3$ & 19 & 3.8 & $1.8-7.6$ \\
\hline Sheetmetal workers (NYK 753) & 184 & 1.5 & $1.3-1.8$ & 48 & 1.8 & $1.3-2.4$ & 3 & 3.6 & $0.9-14.5$ & 2 & 2.5 & $0.6-10.4$ \\
\hline Plumbers, pipefitters (NYK 754) & 125 & 1.2 & $1.0-1.4$ & 27 & 1.5 & $1.0-2.2$ & - & .. &.. & 1 & 9.8 & $1.4-69.2$ \\
\hline Welders (NYK 755) & 287 & 2.7 & $2.3-3.1$ & 69 & 2.9 & $2.1-4.1$ & 5 & 1.6 & $0.6-4.4$ & 4 & 3.2 & $1.0-10.3$ \\
\hline Metal platers and coaters (NYK 757) & 20 & 2.9 & $1.7-4.8$ & 6 & 1.8 & $0.7-4.8$ & - &.. &.. & - & .. & .. \\
\hline Construction carpenters (NYK 771) & 247 & 1.0 & $0.9-1.1$ & 86 & 2.5 & $1.9-3.1$ & - & .. & .. & 2 & 97.5 & $15-636$ \\
\hline Woodworking machine operators (NYK 772) & 173 & 1.7 & $1.4-2.1$ & 90 & 2.7 & $2.1-3.6$ & 15 & 11.3 & $4.4-29.5$ & 15 & 7.3 & $3.0-18.0$ \\
\hline Painters (NYK 781) & 248 & 1.8 & $1.6-2.0$ & 53 & 2.9 & $2.0-4.2$ & 4 & 4.2 & $1.1-16.6$ & 1 & 0.3 & $0.05-2.4$ \\
\hline Bricklayers (NYK 791) & 83 & 2.2 & $1.7-2.8$ & 10 & 1.7 & $0.8-3.6$ & - & .. &. & - & .. & .. \\
\hline Concrete and construction workers (NYK 793) & 259 & 1.6 & $1.4-1.8$ & 115 & 2.0 & $1.6-2.4$ & - & .. & .. & - & .. & .. \\
\hline Divers (NYK 797) & 38 & 1.7 & $1.2-2.4$ & 36 & 1.6 & $1.1-2.3$ & - & .. & .. & - & .. & .. \\
\hline Typographers (NYK 801) & 186 & 1.6 & $1.4-2.0$ & 23 & 1.8 & $1.1-3.1$ & 16 & 2.1 & $1.2-3.6$ & 3 & 1.3 & $0.4-4.7$ \\
\hline Bookbinders (NYK 806) & 20 & 1.9 & $1.2-3.1$ & 7 & 6.9 & $2.2-21.2$ & 8 & 2.5 & $1.1-5.5$ & 2 & 2.8 & $0.7-11.4$ \\
\hline Bakers or pastry cooks (NYK 822) & 52 & 3.9 & $1.3-11.6$ & 4 & 0.6 & $0.2-1.7$ & 4 & 1.0 & $0.4-3.0$ & 3 & 10.0 & $2.0-51.2$ \\
\hline Butchers (NYK 826) & 64 & 1.9 & $1.4-2.5$ & 23 & 10.6 & $4.4-25.6$ & 4 & 0.8 & $0.3-2.5$ & 2 & 2.9 & $0.5-16.1$ \\
\hline Paper or paper product makers (NYK 836) & 74 & 2.3 & $1.6-3.4$ & 20 & 1.7 & $0.9-3.5$ & 4 & 2.3 & $0.5-10.2$ & - & .. & .. \\
\hline Plastic product makers (NYK 852) & 40 & 1.4 & $0.9-2.3$ & 27 & 2.7 & $1.0-7.6$ & 13 & 3.9 & $1.8-8.7$ & 8 & 1.4 & $0.6-3.3$ \\
\hline Other production nec (NYK 858) & 36 & 1.8 & $1.2-2.8$ & 24 & 2.0 & $1.2-3.5$ & 7 & 3.6 & $1.2-11.4$ & 3 & 1.1 & $0.3-3.6$ \\
\hline Unskilled manual workers (NYK 861) & 122 & 2.5 & $2.0-3.0$ & 64 & 3.0 & $2.3-4.0$ & - & .. & .. & 2 & 6.8 & $1.2-38.5$ \\
\hline Truck and conveyor operators (NYK 875) & 135 & 1.6 & $1.3-2.0$ & 72 & 2.3 & $1.7-3.0$ & 3 & 1.3 & $0.4-4.2$ & 3 & 2.1 & $0.7-6.9$ \\
\hline Packers (NYK 881) & 46 & 2.2 & $1.6-3.0$ & 34 & 3.0 & $2.0-4.3$ & 14 & 1.1 & $0.6-2.0$ & 21 & 4.0 & $2.0-8.0$ \\
\hline Dockers, freight handlers (NYK 882) & 55 & 2.7 & $1.9-3.8$ & 9 & 2.5 & $1.0-6.1$ & - & .. &.. & - & .. & .. \\
\hline Store and warehouse workers (NYK 883) & 251 & 1.6 & $1.4-1.9$ & 121 & 2.0 & $1.7-2.4$ & 13 & 0.8 & $0.4-1.4$ & 13 & 1.8 & $1.1-3.2$ \\
\hline Cooks (NYK 912) & 59 & 2.8 & $2.0-3.8$ & 18 & 2.6 & $1.5-4.5$ & 48 & 2.4 & $1.8-3.3$ & 22 & 1.7 & $1.1-2.6$ \\
\hline Kitchen assistants (NYK 913) & 24 & 2.0 & $1.0-3.9$ & 20 & 3.9 & $2.3-6.6$ & 80 & 1.9 & $1.5-2.5$ & 59 & 3.5 & $2.6-4.6$ \\
\hline Home helpers (NYK 915) & 6 & 1.9 & $0.8-4.4$ & 31 & 5.0 & $3.1-8.1$ & 87 & 1.8 & $1.5-2.3$ & 167 & 2.0 & $1.7-2.4$ \\
\hline Waiters (NYK 921) & 26 & 1.5 & $0.7-3.2$ & 3 & 4.3 & $0.7-26.0$ & 39 & 1.5 & $1.1-2.2$ & 15 & 1.5 & $0.9-2.6$ \\
\hline Building caretakers (NYK 931) & 268 & 1.7 & $1.5-2.0$ & 166 & 2.1 & $1.8-2.5$ & 4 & 1.6 & $0.5-5.2$ & 6 & 14.1 & $2.5-80$ \\
\hline Cleaners (NYK 932) & 95 & 2.5 & $1.9-3.3$ & 83 & 5.0 & $3.9-6.4$ & 266 & 2.3 & $2.0-2.7$ & 70 & 2.8 & $2.2-3.6$ \\
\hline Hairdressers (NYK 941) & 30 & 1.7 & $1.1-2.5$ & 1 & 1.5 & $0.2-10.8$ & 39 & 1.2 & $0.9-1.6$ & 3 & 1.0 & $0.3-3.0$ \\
\hline
\end{tabular}

a Code of the Nordic modification of the International Classification of Occupations (NYK) in parentheses.

cupations among the women and men were mainly the same. However, contrary to the men, the female social workers had low relative risks, while male social workers had a significantly increased risk among both the persons in stable and those in unstable work. Similarly female child nurses were at low risk, while male child nurses with long-term employment in the occupation had a significantly increased relative risk, although based on only 10 cases. Female drivers showed an increased risk, while male drivers, at least with long-term employment in the occupation, did not have an increased risk. Female policemen showed a significantly increased relative risk, although based on only six cases, while male policemen had a significantly decreased risk.

\section{Liver cirrhosis diagnoses according to occupation}

Among those with long-term employment in the same occupation during 1985-1990, five occupations among the men (messengers, construction carpenters, painters, truck and conveyor operators, store and warehouse workers) and one occupation among the women (cleaners), based on least 20 cases in the occupation, showed 
Table 4. Standardized rate ratios (SRR) for the occupations with increased risk of alcoholism diagnoses among long-term employed men and women (who reported the same occupation in 1985 and 1990) and among newly recruited (ie, short-term employed) workers (those who reported the occupation only in 1990 and reported another occupation in 1985). The reference category was the entire study population (ie, those who reported an occupation in both censuses). Only occupations for which the lower limit of the $95 \%$ confidence interval $(95 \% \mathrm{Cl}$ ) was $\geq 1.0$ based on at least 20 cases among the men (stable or unstable in occupation) or women (stable or unstable in occupation) are listed. SRR values in bold indicate that the criteria was fulfilled. (nec = not elsewhere classified, theoret $=$ theoretical, aesthet $=$ aesthetic, pract $=$ practical $)$

\begin{tabular}{|c|c|c|c|c|c|c|c|c|c|c|c|c|}
\hline \multirow{3}{*}{ Occupation a } & \multicolumn{6}{|c|}{ Men } & \multicolumn{6}{|c|}{ Women } \\
\hline & \multicolumn{3}{|c|}{ Long-term employed } & \multicolumn{3}{|c|}{ Short-term employed } & \multicolumn{3}{|c|}{ Long-term employed } & \multicolumn{3}{|c|}{ Short-term employed } \\
\hline & N & SRR & $95 \% \mathrm{Cl}$ & $\mathrm{N}$ & SRR & $95 \% \mathrm{Cl}$ & $\mathrm{N}$ & SRR & $95 \% \mathrm{Cl}$ & $\mathrm{N}$ & SRR & $95 \% \mathrm{Cl}$ \\
\hline Architects, civil engineers, technicians (NYK 001) & 152 & 0.4 & $0.3-0.5$ & 11 & 0.6 & $0.4-0.8$ & 5 & 0.4 & $0.2-1.1$ & 2 & 0.7 & $0.2-3.0$ \\
\hline Electrical engineers and technicians (NYK 002) & 105 & 0.3 & $0.2-0.4$ & 40 & 0.5 & $0.3-0.7$ & 8 & 1.4 & $0.6-3.0$ & 4 & 1.2 & $0.4-3.3$ \\
\hline Electronic, teletechnical engineers (NYK 003) & 181 & 0.4 & $0.4-0.5$ & 65 & 0.5 & $0.4-0.7$ & 4 & 1.0 & $0.3-2.8$ & 1 & 0.5 & $0.1-3.4$ \\
\hline Engineers, engineering technicians nec (NYK 006) & 54 & 0.6 & $0.4-0.8$ & 33 & 0.8 & $0.4-1.8$ & 2 & 0.6 & $0.1-2.9$ & 2 & 0.6 & $0.1-2.2$ \\
\hline Technical workers nec (NYK 009) & 23 & 0.7 & $0.4-1.4$ & 15 & 0.3 & $0.2-0.5$ & 1 & 0.2 & $0.03-1.5$ & 2 & 0.4 & $0.1-1.8$ \\
\hline Physicians and surgeons (NYK 031) & 29 & 0.2 & $0.1-0.3$ & 2 & 0.5 & $0.1-2.5$ & 5 & 0.3 & $0.1-0.7$ & 1 & 0.5 & $0.1-3.3$ \\
\hline Dentists (NYK 032) & 14 & 0.3 & $0.2-0.5$ & 1 & 0.4 & $0.1-3.0$ & 5 & 0.6 & $0.2-1.7$ & - & .. & .. \\
\hline Registered nurses (NYK 040) & 33 & 1.1 & $0.7-1.8$ & 5 & 1.6 & $0.5-5.3$ & 95 & 0.7 & $0.5-0.8$ & 12 & 0.7 & $0.4-1.3$ \\
\hline Physiotherapists (NYK 047) & 14 & 0.9 & $0.5-1.7$ & 5 & 1.2 & $0.3-4.8$ & 23 & 0.6 & $0.4-0.8$ & 4 & 0.5 & $0.2-1.2$ \\
\hline Principals, headmasters (NYK 050) & 6 & 0.1 & $0.04-0.3$ & 2 & 0.2 & $0.04-0.7$ & 1 & 0.6 & $0.1-4.3$ & - & .. & .. \\
\hline University or higher education teachers (NYK 051) & 21 & 0.3 & $0.2-0.6$ & 5 & 0.4 & $0.1-1.1$ & 2 & 0.2 & $0.04-0.7$ & - & .. & .. \\
\hline Secondary education teachers (theoret) (NYK 052) & 77 & 0.4 & $0.3-0.5$ & 13 & 0.5 & $0.3-1.0$ & 34 & 0.5 & $0.3-0.8$ & 6 & 0.6 & $0.3-1.6$ \\
\hline Primary education teachers (NYK 053) & 34 & 0.5 & $0.4-0.8$ & 3 & 0.3 & $0.08-1.0$ & 49 & 0.3 & $0.3-0.5$ & 7 & 2.3 & $0.7-7.8$ \\
\hline Secondary education t. (aesthet, pract) (NYK 054) & 36 & 0.6 & $0.4-0.9$ & 5 & 1.9 & $0.4-9.6$ & 12 & 0.3 & $0.2-0.6$ & 2 & 0.9 & $0.2-4.7$ \\
\hline Technical education teachers (NYK 055) & 29 & 0.5 & $0.3-0.7$ & 7 & 0.7 & $0.3-1.5$ & 1 & 0.2 & $0.02-1.1$ & 2 & 1.0 & $0.2-4.0$ \\
\hline Preprimary education teachers (NYK 056) & 4 & 0.3 & $0.1-0.7$ & 2 & 0.2 & $0.05-0.8$ & 22 & 0.2 & $0.1-0.3$ & 4 & 0.2 & $0.07-0.6$ \\
\hline Ministers, priests (NYK 061) & 3 & 0.1 & $0.03-0.3$ & 1 & 0.5 & $0.07-3.7$ & 1 & 0.6 & $0.1-4.5$ & - & .. & .. \\
\hline Auditors (NYK 091) & 19 & 0.5 & $0.3-0.8$ & 2 & 0.2 & $0.05-0.9$ & 3 & 0.6 & $0.2-1.9$ & - & .. & .. \\
\hline Social workers (NYK 092) & 72 & 1.3 & $1.0-1.7$ & 31 & 1.5 & $1.0-2.2$ & 32 & 0.5 & $0.3-0.7$ & 15 & 0.6 & $0.4-1.1$ \\
\hline Economists, statisticians (NYK 094) & 40 & 0.5 & $0.3-0.9$ & 15 & 0.6 & $0.3-1.2$ & 10 & 0.7 & $0.3-1.5$ & 11 & 0.7 & $0.4-1.4$ \\
\hline Personal officers (NYK 096) & 58 & 0.7 & $0.5-0.9$ & 19 & 0.4 & $0.2-0.6$ & 25 & 0.8 & $0.5-1.4$ & 11 & 0.5 & $0.3-1.0$ \\
\hline System analysts, programmers (NYK 097) & 11 & 0.2 & $0.1-0.2$ & 10 & 0.3 & $0.1-0.7$ & 3 & 0.4 & $0.1-1.9$ & 6 & 0.4 & $0.2-1.0$ \\
\hline Public administrators (government) (NYK 101) & 49 & 0.3 & $0.2-0.5$ & 28 & 0.4 & $0.3-0.7$ & 11 & 0.6 & $0.2-1.5$ & 23 & 0.6 & $0.4-0.9$ \\
\hline Business administrators (NYK 111) & 40 & 0.4 & $0.3-0.7$ & 36 & 0.4 & $0.3-0.7$ & 3 & 1.4 & $0.4-4.9$ & 3 & 0.8 & $0.2-2.4$ \\
\hline Business administrative workers nec (NYK 118) & 52 & 0.3 & $0.2-0.4$ & 29 & 0.3 & $0.2-0.5$ & 26 & 1.0 & $0.6-1.6$ & 13 & 0.4 & $0.2-0.8$ \\
\hline Bookkeeping and cashier's work (NYK 201) & 26 & 0.8 & $0.5-1.3$ & 12 & 1.0 & $0.5-1.8$ & 35 & 0.6 & $0.4-0.9$ & 21 & 0.7 & $0.5-1.8$ \\
\hline Bank employees (NYK 292) & 15 & 0.3 & $0.2-0.6$ & 2 & 0.3 & $0.07-1.5$ & 15 & 0.3 & $0.2-0.5$ & 1 & 0.2 & $0.03-1.3$ \\
\hline Property managers (NYK 295) & 49 & 0.8 & $0.5-1.1$ & 32 & 0.5 & $0.4-0.8$ & - & .. & .. & 1 & 0.2 & $0.03-1.7$ \\
\hline Insurance clerks (NYK 296) & 10 & 0.4 & $0.1-1.0$ & 2 & 0.2 & $0.06-1.0$ & 12 & 0.7 & $0.3-1.4$ & 3 & 0.6 & $0.2-2.5$ \\
\hline Working proprietors, wholesale (NYK 301) & 20 & 0.6 & $0.4-1.0$ & 33 & 0.7 & $0.5-1.1$ & - & .. & .. & 4 & 1.2 & $0.4-3.6$ \\
\hline Advertising salesmen (NYK 313) & 29 & 0.6 & $0.3-1.1$ & 16 & 0.3 & $0.2-0.5$ & 4 & 0.3 & $0.1-0.8$ & 2 & 0.2 & $0.04-0.8$ \\
\hline Buyers (NYK 331) & 243 & 0.5 & $0.4-0.6$ & 69 & 0.4 & $0.3-0.6$ & 23 & 0.7 & $0.6-1.1$ & 11 & 0.4 & $0.2-0.7$ \\
\hline Shop managers (NYK 332) & 28 & 0.6 & $0.4-0.9$ & 17 & 0.7 & $0.4-1.1$ & 6 & 1.0 & $0.4-2.5$ & 12 & 2.9 & $1.3-6.2$ \\
\hline Working proprietors, agriculture/forestry (NYK 401) & 88 & 0.5 & $0.3-1.0$ & 20 & 0.7 & $0.3-1.4$ & 4 & 0.01 & $.003-0.02$ & 2 & 4.1 & $0.6-27.1$ \\
\hline Forestry managers (NYK 403) & 4 & 0.2 & $0.1-0.5$ & 2 & 0.7 & $0.2-2.9$ & _- &.. & .. & - & .. & .. \\
\hline Agricultural workers (NYK 411) & 8 & 0.2 & $0.1-0.5$ & 9 & 1.4 & $0.4-4.5$ & 1 & 0.1 & $0.02-0.9$ & 1 & 0.4 & $0.05-2.6$ \\
\hline Railway engine drivers (NYK 631) & 12 & 0.3 & $0.2-0.6$ & 5 & 0.6 & $0.2-1.5$ & - & .. & .. & - & .. & .. \\
\hline Road transport supervisors (NYK 644) & 15 & 0.5 & $0.3-0.9$ & 17 & 0.9 & $0.5-1.5$ & 1 & 2.3 & $0.3-16.4$ & _- & _- & _- \\
\hline Firefighters (NYK 901) & 28 & 0.6 & $0.4-0.9$ & - & .. & .. & - & .. & .. & - & .. & .. \\
\hline Policemen (NYK 902) & 56 & 0.4 & $0.3-0.6$ & 2 & 0.5 & $0.09-2.6$ & 6 & 2.8 & $1.1-7.4$ & _- & .. & .. \\
\hline Civilian protective service workers nec (NYK 908) & 30 & 0.7 & $0.5-1.0$ & 14 & 1.4 & $0.8-2.4$ & 5 & 1.4 & $0.5-3.9$ & 3 & 1.2 & $0.4-3.8$ \\
\hline Children nurses (NYK 914) & 10 & 3.9 & $1.5-10.4$ & 4 & 0.7 & $0.3-2.1$ & 60 & 0.4 & $0.3-0.6$ & 35 & 1.2 & $0.8-1.8$ \\
\hline Photographers (NYK 946) & 16 & 0.5 & $0.3-0.8$ & 1 & 0.1 & $0.01-0.8$ & 1 & 2.0 & $0.3-14.5$ & 1 & 0.9 & $0.1-6.5$ \\
\hline Officers (armed forces) (NYK 981) & 53 & 0.5 & $0.4-0.7$ & 5 & 0.6 & $0.2-1.8$ & - & .. & .. & - & .. & .. \\
\hline
\end{tabular}

${ }^{a}$ Code of the Nordic modification of the International Classification of Occupations (NYK) in parentheses.

increased relative risks of liver cirrhosis as diagnosed in inpatient care (data not shown).

\section{Alcohol-related mortality according to occupation}

Among those who were stable in the same occupation during 1985-1990, 13 occupations among the men (assistant nurses, secretaries, horticultural workers, forest workers, messengers, toolmakers, machinery fitters, sheetmetal workers, welders, concrete and construction workers, unskilled manual workers, truck and conveyor operators, store and warehouse workers) and two occupations among the women (secretaries, cleaners), based on least 20 cases in the occupation, showed increased relative risks of alcohol-related mortality (data not shown). 


\section{Discussion}

Among the persons with long-term employment, several occupations had increased relative risks of alcoholism diagnoses. New recruits to such occupations also often had even more increased relative risks of alcoholism; this finding indicates that heavy drinkers are selected to such occupations. Although based on fewer cases, the pattern concerning occupational differences in liver cirrhosis diagnosed in inpatient care and alcohol-related mortality seems to be the same as for alcohol diagnoses.

The pattern concerning occupational differences in alcoholism diagnoses among the women resembled that among the men.

\section{Misclassification of outcome data}

A differential misclassification of outcome would occur if persons from lower social strata, given comparable symptomatology, more easily receive an alcoholism diagnosis than persons from higher social strata. It has been suggested that such misclassification does occur, for example, due to the attitudes of physicians (12). However, such misclassification is probably limited.

First, if such differential misclassification of outcome occurs, it is more likely that it occurs in inpatient care, where the patient is present, and than in diagnoses at death. However, socioeconomic differences in alcohol-related mortality in Sweden show the same pattern as for alcoholism diagnoses in inpatient care.

Second, studies from other countries, with a similar socioeconomic pattern for alcohol-related mortality, show that the risk use of alcohol and alcohol dependence are more common among manual workers (6). The Swedish Lundby study also reported similar findings in that a higher proportion of manual workers than other groups was found to meet the criterion of alcohol abuse and dependence (13).

\section{Long-term and short-term employment}

In this study stable work (long-term employment) was measured as a report of the same occupation in the censuses in 1985 and 1990. Those who reported being in a specific occupation in 1990 and who had reported another occupation in 1985 were considered to be new recruits (short-term employment) into the occupation in 1990. This group was likely to have stayed in an occupation much less than five years on the average. Both categories were likely to have been misclassified. The misclassification is likely to have been nondifferential, and the association between each category and alcoholism would have been underestimated. According to the results of this study new recruits and stable workers often had increased relative risks of the same magnitude.
We did not find a reason for assuming that one category (new recruits versus persons with long-term employment) should be more misclassified than the other.

\section{Is there a role for selection?}

Plant (14) found that recruits to an occupation at high risk of alcoholism (ie, brewery workers in Scotland) were more often heavy drinkers than recruits to other occupations. Moreover, persons who disliked heavy drinking tended to leave the occupation. He also reported that the drinking behavior of workmates could increase individual drinking and encourage problemdrinking (14). Such findings indicate that tolerance towards alcohol consumption in the workplace can be regarded as a risk factor in itself, especially for persons with some kind of predisposition to alcohol problems. If an increased risk of alcoholism in an occupation can partly be due to a process of selection, it is likely that the following two conditions exist: (i) persons prone to alcohol misuse are more likely to be recruited into the occupation than persons not prone to misuse and (ii) there is tolerance (from employers and workmates) towards the employment of persons prone to alcohol misuse. If only the second is true, but not the first, we would conclude that selection is of minor importance.

Three combinations of long-term or short-term employment and an increased risk of alcoholism diagnosis were found in this study. First, if persons employed long-term in some occupations show an increased risk of alcoholism and those newly recruited do not, circumstances within the occupation are probably important for the development of alcoholism. This pattern was more or less seen for only a few occupations among the men in this study (eg, long-term bakers had a significantly increased relative risk of 3.5 and those newly recruited had a relative risk of 0.6 ).

Second, if an increased risk is found among both persons with long-term employment and those newly recruited to the same occupation, as for most high-risk occupations in this study, selection probably plays a role. In this study we showed that the pattern concerning relative risks in occupations among new recruits, using the same reference category, was very similar to that among persons in stable work in the same occupation. However, the newly recruited employees in each occupation generally showed somewhat higher relative risks than the stable group. This finding is in agreement with the results of the Plant study (14) and indicates that people who enter high-risk occupations are, on the average, more prone to problem drinking than those who are stable in their occupation. Our interpretation is that the first group of persons has often developed drinking problems already before entering the particular 
occupation and that selection is of some importance for occupational variation in alcoholism.

Third, we found two occupations in which the persons with long-term employment did not show an increased relative risk while those newly recruited did (drivers, construction carpenters). Some occupations may accept persons with high alcohol consumption but not persons who develop signs of alcohol abuse.

\section{Comparison with previous studies on occupation and alcoholism}

The following occupations with an elevated risk of alcoholism in this study have previously been associated with a high risk of alcoholism in studies from different countries (mainly Great Britain and the United States) among men: seamen $(1,3,7,15,16,18-20)$, construction workers $(3-5,7)$, painters $(3)$, bricklayers $(7)$, sheetmetal workers $(7)$, and waiters $(2,7)$. Among women an increased risk has been reported for waitresses (5, 7).

We previously investigated alcoholism diagnosis at the time of discharge from inpatient care in Sweden in 1981-1983 among persons who held the same occupation in the censuses of 1960 and 1970 (8). Also in this study, occupations with increased relative risks were mostly manual, and occupations with low risks were nonmanual. In these previous analyses 26 occupations were found to have increased relative risks for alcohol diagnoses in inpatient care. Among the men, five occupations with increased risk in the previous investigation did not show any risk increase in our follow-up for the years 1991-1994 (journalists, photographers, dental technicians, advertising salesmen, shop assistants). For some occupations a change from high to low or average risk could have been due to a change in the tolerance of employers and workmates towards heavy alcohol use among employees.

\section{Alcoholism diagnoses in male- and female-dominated occupations}

During the 1970s female labor market participation increased, and, at the end of the 1980s, it was as high as for men. While alcohol consumption among women increased during the 1950s and 1960s, this trend has not continued since then in Sweden (21). According to the results of our study, it seems as if women in some maledominated, mainly blue-collar, occupations have an increased relative risk of developing alcohol problems [eg, drivers, toolmakers, machinery fitters, and, with less than 20 cases, horticultural workers, woodworking machine operators, painters, typographers, bookbinders, plastics (and other) product makers and police]. This trend could be due to the fact that women in male-dom- inated occupations adapt to male drinking behavior. It has also been suggested that people of the sexual minority in a workplace are under particular stress in their worklife $(22,23)$; this stress is possibly relieved by increased alcohol consumption. We also found that men in some female-dominated occupations have an increased relative risk of alcoholism diagnoses, namely, assistant nurses, home helpers, and cleaners.

\section{Concluding remarks}

In this study manual workers showed the highest relative risk of alcoholism. The increased relative risks of alcoholism diagnoses found for several, mostly manual, occupations seemed partly to be an effect of the selection of heavy drinkers into the same occupations. This conclusion is based on the evidence that new recruits into high-risk occupations often had increased relative risks of at least the same magnitude as those stable in the occupation over several years.

The pattern concerning relative risk differences in alcoholism between occupations among women was similar to that among men, although with lower absolute risks.

\section{Acknowledgments}

The authors wish to thank Ingvar Lundberg for his comments on an earlier version of the paper.

\section{References}

1. Plant MA. Alcoholism and occupation: a review. Br J Addict 1977;72:309-16.

2. Fillmore K, Caetano R. Epidemiology of alcohol abuse and alcoholism in occupations. In: Occupational alcoholism: a review of research issues. Washington (DC): US Government Printing Office, 1982. DHHS publication (ADM), NIAAA research monograph no 8.

3. Olkinuora M. Alcoholism and occupation. Scand J Work Environ Health 1984;10:11-5.

4. Harford TC, Brooks SD. Cirrhoses mortality and occupation. J Stud Alcohol 1992;53:463-8

5. Mandell W, Eaton WW, Anthony JC, Garrison R. Alcoholism and occupations: a review and analysis of 104 occupations. Alcohol Clin Exp Res 1992;16:734-46.

6. Parker DA, Harford TC. The epidemiology of alcohol consumption and dependence across occupations in the United States. Alcohol Health Res W 1992;16:97-105.

7. Leigh JP, Jiang WY. Liver cirrhoses deaths within occupations and industries in the California occupational mortality study. Addiction 1993;84:767-79.

8. Hemmingsson T, Lundberg I, Romelsjö A, Alfredsson L. Alcoholism in social classes and occupations in Sweden. Int J 
Epidemiol 1997;26:584-91.

9. Martin JK, Roman PM, Blum TC. Job stress, drinking networks, and social support at work: a comprehensive model of employees' problem drinking behaviors. Sociol Q 1996; 37:579-99.

10. Von Wiegand RA. Alcoholism in industry. Br J Addict 1972;67:181-7.

11. National Board of Health and Welfare, Center for Epidemiology. Statistics — health and diseases: causes of death 1995. Stockholm: SCB, 1996. Official Statistics of Sweden.

12. Wolf I, Morris IC, Blane HT, Hill MJ. Social factors in the diagnosis of alcoholism, II: attitudes of physicians. Q J Stud Alcohol 1965;26:72-9.

13. Öjesjö L. The relationship to alcoholism of occupation, class and employment. J Occup Med 1980;2:657-66.

14. Plant MA. Occupations and alcoholism: cause or effect? A controlled study of recruits to the drink trade. Int J Addict 1978;13:605-26.

15. Åmark C. A study of alcoholism. Acta Psychiatr Neurol Scand 1951;suppl 70.

16. Ågren G, Romelsjö A. Mortality in alcohol-related diseases in Sweden during 1971-80 in relation to occupation, marital status and citizenship in 1970. Scand J Soc Med 1992;20; 134-42.
17. Slattery M, Alderson MR, Bryant JS. The occupational risks of alcohol. Int J Addict 1986;21:929-36.

18. Whitehead PC, Simpkins J. Occupational factors in alcoholism. In: Kissin B, Begleiter H, editors. The pathogenesis of alcoholism: psychosocial factors. New York (NY): Plenum Press, 1983.

19. Hitz D. Drunken sailors and others: drinking problems in specific occupations. Q J Stud Alcohol 1973;34:496-505.

20. Murray RM. Alcoholism and employment. J Alcohol 1974; 10:23-6.

21. Kühlhorn E. Problem och nya grepp i kartläggningar av alkoholkonsumtionen och alkoholskadorna [in Swedish]. In: Kühlhorn E, Björ J, editors. Svenska alkoholvanor i förändring. Kristianstad: Sober förlag, 1998.

22. Hunt K, Emslie C. Men's work, women's work: is there an effect of taking account of occupational sex differences in health? In: Orth-Gomer K, Chesney M, Wenger N, editors. Stress and heart disease. Hillsdale (NJ): Lawrence Erlbaum, 1997.

23. Östlin P, Alfredsson L, Hammar N, Reuterwall C. Myocardial infarction in male and female dominated occupations. Occup Environ Med 1998;55:642-4.

Received for publication: 18 December 2000 